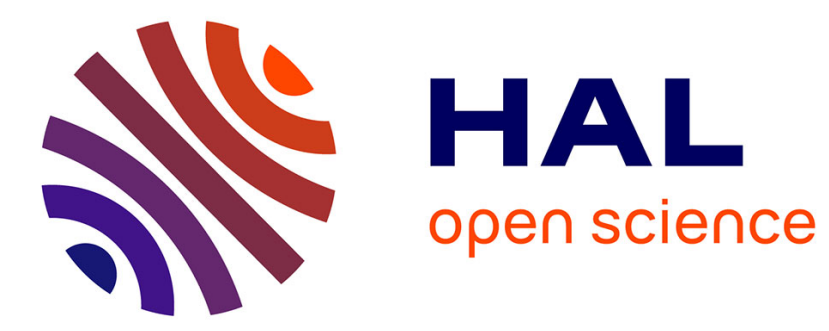

\title{
Time-resolved vibrations of two-dimensional hypersonic phononic crystals
}

\author{
J.F. Robillard, Arnaud Devos, I. Roch-Jeune
}

\section{To cite this version:}

J.F. Robillard, Arnaud Devos, I. Roch-Jeune. Time-resolved vibrations of two-dimensional hypersonic phononic crystals. Physical Review B: Condensed Matter and Materials Physics (1998-2015), 2007, 76, pp.092301. 10.1103/PhysRevB.76.092301 . hal-00255742

\section{HAL Id: hal-00255742 \\ https://hal.science/hal-00255742}

Submitted on 27 Jul 2021

HAL is a multi-disciplinary open access archive for the deposit and dissemination of scientific research documents, whether they are published or not. The documents may come from teaching and research institutions in France or abroad, or from public or private research centers.
L'archive ouverte pluridisciplinaire HAL, est destinée au dépôt et à la diffusion de documents scientifiques de niveau recherche, publiés ou non, émanant des établissements d'enseignement et de recherche français ou étrangers, des laboratoires publics ou privés. 


\title{
Time-resolved vibrations of two-dimensional hypersonic phononic crystals
}

\author{
J.-F. Robillard, A. Devos,* and I. Roch-Jeune \\ Institut d'Électronique, de Microélectronique et de Nanotechnologie, Unité Mixte de Recherche, CNRS 8520, \\ Avenue Poincaré Boîte Postale 69, F-59652 Villeneuve d'Ascq Cedex, France \\ (Received 13 July 2007; revised manuscript received 3 August 2007; published 11 September 2007)
}

\begin{abstract}
We fabricate e-beam lithographied two-dimensional lattices of nanometric metal cubes of various sizes and lattice parameters. Using ultrafast laser acoustics, we time resolve two kinds of vibrations in such crystals: individual cube vibrations and lower frequencies strongly dependent on the lattice parameter. From complementary experiments and an analytical model, we assign these modes to acoustic collective vibrations (i.e., acoustic phonons) of the artificial crystal. Their unprecedented detection takes advantage of another periodicity effect acting on the optical field.
\end{abstract}

DOI: 10.1103/PhysRevB.76.092301

PACS number(s): 63.22.+m, 63.20.Dj, 78.20.Hp, 78.47.+p

Any wave which propagates in a periodic structure whose lattice parameter is comparable to the wavelength experiences similar "band" effects. ${ }^{1}$ In that sense, there are analogies between electronic states in a crystal or light modes in a photonic crystal, the most common being the apparition of forbidden energies also called band gaps. Elastic waves are also expected to experience such periodic effects in so-called phononic crystals (PCs). The perspective of designing an on demand material by adjusting the lattice type and parameter has recently motivated numerous studies on PC. ${ }^{2,3}$ Most applications of PC have focused on low frequency ranges due to technological constraints. In order to reach the hypersonic range (i.e., beyond $1 \mathrm{GHz}$ ), the lattice constant must be between a few $100 \mathrm{~nm}$ and a few microns. Such crystals are both phononic and photonic, from which new effects are expected, in particular, concerning the acousto-optic interaction. $^{4}$

Basically, a hypersonic PC is made of a periodically repeated nanoscaled pattern. The vibration modes of the isolated pattern lead to branches in the crystal due to the coupling between structures. As in a molecular crystal, in the case of a weak coupling such branches are almost flat around the isolated frequencies. Much lower frequency modes exist only in the crystal. They correspond to translations of the rigid pattern from the equilibrium position. These second kind of modes are similar to acoustic-phonon branches in any crystal. Close to zero, the wave vector and the frequency are proportional to a factor equal to the sound velocity. Contrary to the first type, the frequencies of these modes are strongly dependent on the lattice.

Acoustic phonons in nanostructures have been investigated both in the time domain and using Brillouin or Raman spectroscopy. Most of the past studies have reported the observation of individual modes of various nanostructures: nanospheres, ${ }^{5,6}$ nanoparticles, ${ }^{7-12}$ or engineered objects. ${ }^{13,14}$ A few studies have presented acoustic vibrations of periodic structures: surface waves time resolved in micronic structures $^{15,16}$ and phonon modes in submicronic PC probed by Brillouin light scattering. ${ }^{4}$ Until now, collective modes have never been observed in the time domain.

In this Brief Report, we report on a time-resolved study of acoustic modes in two-dimensional (2D) hypersonic PC made of square lattices of nanometric cubes. We demonstrate the coexistence of two kinds of vibrations. We show that the first modes are those of the individual cube. Other modes present a dependence on the step size and cube size, which allow us to assign them to collective modes, reported here in a time-resolved study. We show that these modes have a specific detection mechanism, and we discuss the possible role of photonics or plasmonics in our samples.

We fabricate 2D square lattices of aluminum (Al) parallelepipeds using electronic lithography. This technique enables designing patterns of various shapes and sizes with a precision down to a few nanometers. This way we obtain defect-free samples without dispersion of the dot size $(d)$ or variation of the lattice constant $(a)$. The e-beam prints directly in a positive photoresist and the dots are formed by lift-off of an evaporated Al layer. All these lattices lie on a $100 \mathrm{~nm}$ thick Al film deposited on a Pyrex substrate. A scanning electron microscope (SEM) image of a $200 \mathrm{~nm}$ cubes sample is shown in Fig. 1.

All the studied samples are listed in Table I. Some samples are made of homothetic 50, 100, and $200 \mathrm{~nm}$ sized cubes with various step sizes. The other samples are made of $100 \mathrm{~nm}$ thick Al squares of 400 or $500 \mathrm{~nm}$ side.

The time-resolved experiments were conducted using a one- or two-color pump and probe setup associated with a tunable Ti:sapphire oscillator. The laser produces $120 \mathrm{fs}$ optical pulses at a repetition rate of $76 \mathrm{MHz}$, centered at a wavelength adjustable between 700 and $990 \mathrm{~nm}$. In the twocolor configuration, the probe is frequency doubled to produce a blue pulse. The spot size of laser beams on the sample are about $50 \mu \mathrm{m}$ in diameter. All the experiments are performed at room temperature.
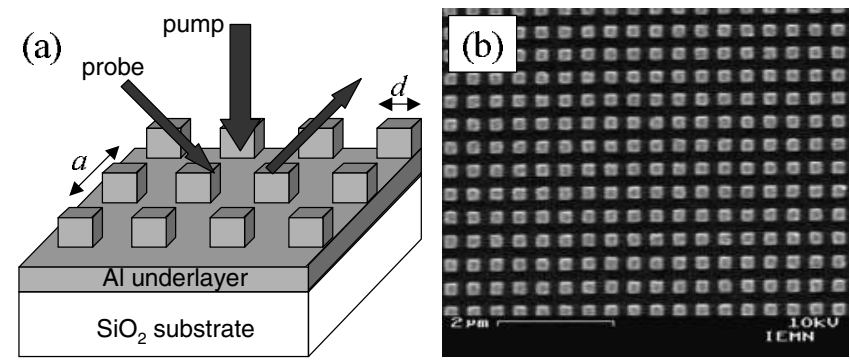

FIG. 1. (a) Schematic diagram of the samples. (b) SEM image of a $d=200 \mathrm{~nm}$ cube, $a=400 \mathrm{~nm}$ step size sample. 
TABLE I. Sample list. The samples marked "c" ("s") are cubes (100 $\mathrm{nm}$ thick squares). The last two columns give $\lambda / a$ for a red and a blue probe wavelength $\lambda$. An asterisk appears when type II modes have been observed.

\begin{tabular}{lccccc}
\hline \hline Sample & Shape & $\begin{array}{c}\text { Size } d \\
(\mathrm{~nm})\end{array}$ & $\begin{array}{c}\text { Step } a \\
(\mathrm{~nm})\end{array}$ & $\begin{array}{c}\lambda / a \\
(800 \mathrm{~nm})\end{array}$ & $\begin{array}{c}\lambda / a \\
(400 \mathrm{~nm})\end{array}$ \\
\hline $\mathrm{A}$ & $\mathrm{c}$ & 50 & 100 & 8.00 & 4.00 \\
$\mathrm{~B}$ & $\mathrm{c}$ & 100 & 200 & 4.00 & 2.00 \\
$\mathrm{C}$ & $\mathrm{c}$ & 200 & 400 & 2.00 & $1.00^{*}$ \\
$\mathrm{D}$ & $\mathrm{c}$ & 50 & 150 & 5.33 & 2.66 \\
$\mathrm{E}$ & $\mathrm{c}$ & 100 & 400 & 2.00 & $1.00^{*}$ \\
$\mathrm{~F}$ & $\mathrm{~s}$ & 400 & 900 & $0.89^{*}$ & $0.44^{*}$ \\
$\mathrm{G}$ & $\mathrm{S}$ & 400 & 1400 & $0.57^{*}$ & $0.28^{*}$ \\
$\mathrm{H}$ & $\mathrm{S}$ & 500 & 1000 & $0.80^{*}$ & $0.40^{*}$ \\
$\mathrm{I}$ & $\mathrm{c}$ & 200 & 450 & 1.78 & $0.89^{*}$ \\
$\mathrm{~J}$ & $\mathrm{c}$ & 200 & 500 & 1.60 & $0.80^{*}$ \\
$\mathrm{~K}$ & $\mathrm{c}$ & 200 & 600 & 1.33 & $0.67^{*}$ \\
$\mathrm{~L}$ & $\mathrm{c}$ & 200 & 700 & $1.14^{*}$ & $0.57^{*}$ \\
$\mathrm{M}$ & $\mathrm{c}$ & 200 & 800 & $1.00^{*}$ & $0.50^{*}$ \\
$\mathrm{~N}$ & $\mathrm{c}$ & 200 & 1000 & $0.80^{*}$ & $0.40^{*}$ \\
$\mathrm{O}$ & $\mathrm{c}$ & 200 & 1200 & $0.67^{*}$ & $0.33^{*}$ \\
\hline \hline
\end{tabular}

We first focus on the cubic shape homothetic samples (samples A-C), in which both size and spacing are proportional. The signals obtained using a pump and a probe both centered at $905 \mathrm{~nm}$ are presented in Fig. 2(a). The transient reflectivity exhibits a sudden change at zero time delay,
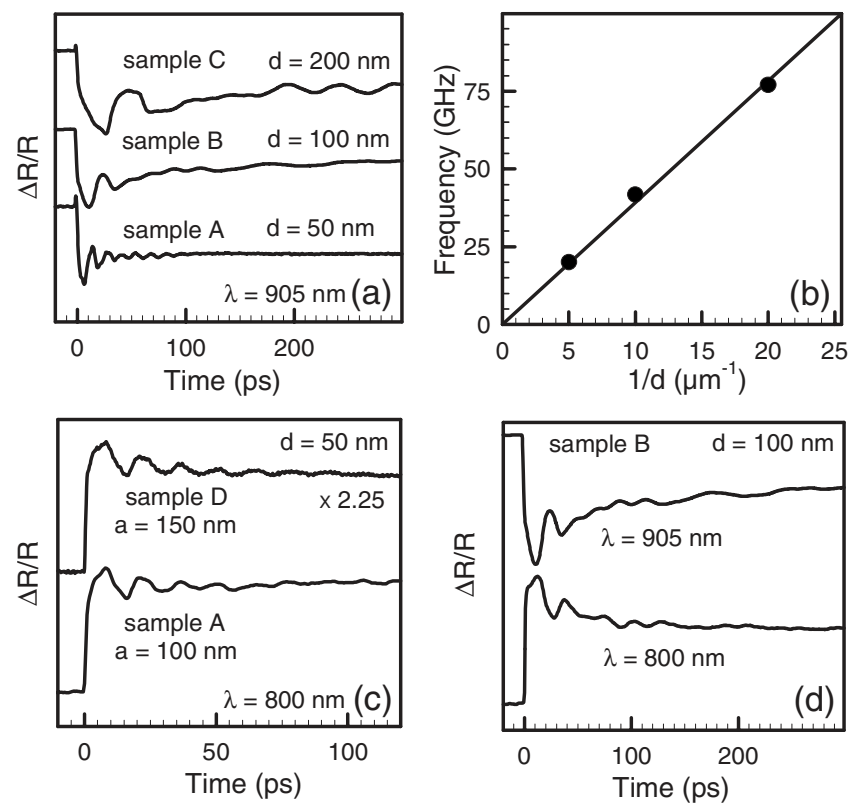

FIG. 2. (a) Transient reflectivity of $d=50,100$, and $200 \mathrm{~nm}$ sized cube lattices measured at $905 \mathrm{~nm}$. (b) The period of the observed oscillation is proportional to the cube size. (c) Signals at $800 \mathrm{~nm}$ from two $d=50 \mathrm{~nm}$ cube samples with different lattice parameters $a$ (samples A and D). (d) Sample B transient reflectivity measured at 800 and $905 \mathrm{~nm}$.
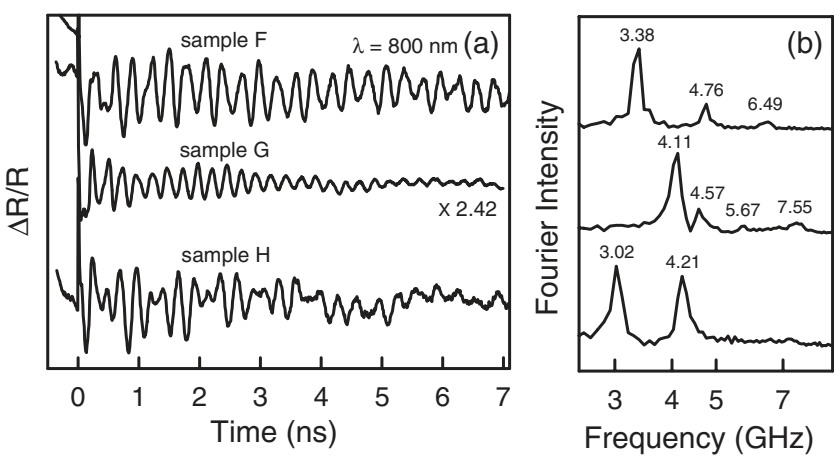

FIG. 3. (a) Transient reflectivity of F, G, and $\mathrm{H}$ samples obtained with an $800 \mathrm{~nm}$ probe. (b) Fourier transform.

which relaxes on a nanosecond time scale. Superimposed on this base signal, we notice strongly damped oscillations whose period is found to increase linearly with the dot size [as shown in Fig. 2(b)]. This is the size dependence expected for the normal modes of any elastic structure. ${ }^{17} \mathrm{We}$, thus, assign these oscillations to the dot vibrations.

Two other arguments support this interpretation. First, the signals were found to be independent of the step size. Figure 2(c) presents the results obtained at $800 \mathrm{~nm}$ on samples A and $\mathrm{D}$, which are both lattices of $50 \mathrm{~nm}$ cubes but with different step sizes $a$. The curve shape and the period measured are identical for both samples. Second, Fig. 2(c) also shows that the signal amplitude is proportional to the dot density [the signal obtained on sample D on Fig. 2(c) has been rescaled to be comparable to those obtained on sample A].

A sign change in the reflectivity of samples A-E is observed when the laser is tuned from 800 to $905 \mathrm{~nm}$. This is visible for sample B in Fig. 2(d). This sign change is an evidence of the photoelastic detection of the vibrations. Indeed, the detection of acoustic contributions in metals is usually governed by the photoelastic couplings. Previous works have shown that these couplings strongly depend on the optical wavelength, especially in the vicinity of interband transitions. ${ }^{18}$ One of us has reported a sign change in $\mathrm{Al}$ detection around $850 \mathrm{~nm}$, which well accounts for the present observation and demonstrates that the cube vibrations are detected through the photoelastic mechanism in Al. ${ }^{19}$

In spite of the very narrow size distribution, a strong damping is observed as expected since the dots and the underlayer are both composed of $\mathrm{Al}$ and, furthermore, the acoustic contrast between the underlayer and the substrate is small.

We obtained strongly different results on the next sample set (samples F-H, middle part of Table I). Figure 3(a) presents the corresponding transient reflectivities at $800 \mathrm{~nm}$. There are major differences compared to the previous results. First point to notice, the oscillations last for at least $8 \mathrm{~ns}$ (note the change in the time scale). Second, the signals have a much higher amplitude and a much lower damping rate.

The Fourier transform visible on Fig. 3(b) exhibits several sharp peaks for each sample. The frequency content here is lower and more intricate than in previous experiments. The most noticeable discrepancy with results obtained in samples 

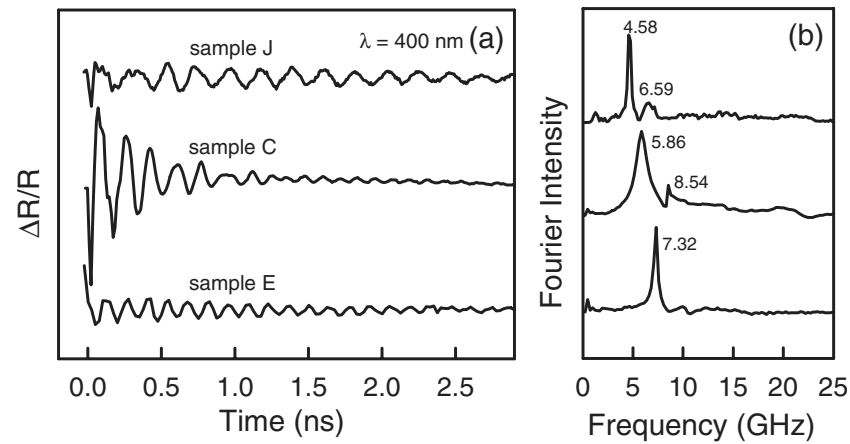

FIG. 4. Transient reflectivity of samples C, E, and J obtained using a blue probe. Samples $\mathrm{C}$ and $\mathrm{J}$ have the same dot size but different lattice parameters. Samples C and E have different dot sizes but identical lattice parameters.

A-E is the dependence of the frequencies on the lattice parameter. This is visible in Fig. 3, on which the results obtained on samples F and $\mathrm{G}$, both composed of $400 \mathrm{~nm}$ dots but with different lattice parameters, are compared.

One can note that the amplitude of the signal is not proportional to the number of dots per unit of surface. This indicates that the collected signal cannot come only from the dots. We did not observe any sign change in the reflectivity tuning the laser from 800 to $905 \mathrm{~nm}$, which attests that the $\mathrm{Al}$ photoelastic detection is not involved in that case.

From all these results we identify two different vibrational phenomena, hereafter labeled as type I and type II. Type I is clearly identified as the individual cube vibrations coherently excited and detected via photoelastic couplings. This observation is similar to previous works on individual vibrations of various nanostructures. Type II modes are also related to the dots, but the most noticeable point is their dependence on the lattice constant. This suggests that type II modes present a collective character resulting from the interdot coupling. Moreover, the signals are qualitatively different, which requires us to investigate another detection mechanism. The rest of the Brief Report is devoted to this last kind of oscillation.

Up to now, the two types of oscillations have been presented on separate samples. By changing the probe wavelength, it is possible to detect both kinds of oscillations in some cubic samples. Figure 4 compares the results obtained on samples C, E, and $\mathrm{J}$ when probed at $400 \mathrm{~nm}$. The use of a blue probe gives a radically different transient reflectivity. In the first $400 \mathrm{ps}$, we retrieve the type I oscillations which are identical to those observed with a red-infrared probe (20 GHz peak on the Fourier transform). The most noticeable point is the detection of strong oscillations which present the characteristics of the type II modes. First, the oscillation is more intense and lasts on the nanosecond time scale. Second, the frequency content is lower and the Fourier transform exhibits two sharp peaks. The frequencies obtained in the three samples are different, which demonstrates that the type II modes do depend on the dot size (samples C and $\mathrm{E}$ have the same lattice parameter but different dot size) and on the lattice parameter (samples $\mathrm{C}$ and $\mathrm{J}$ are both composed of $200 \mathrm{~nm}$ size dots).

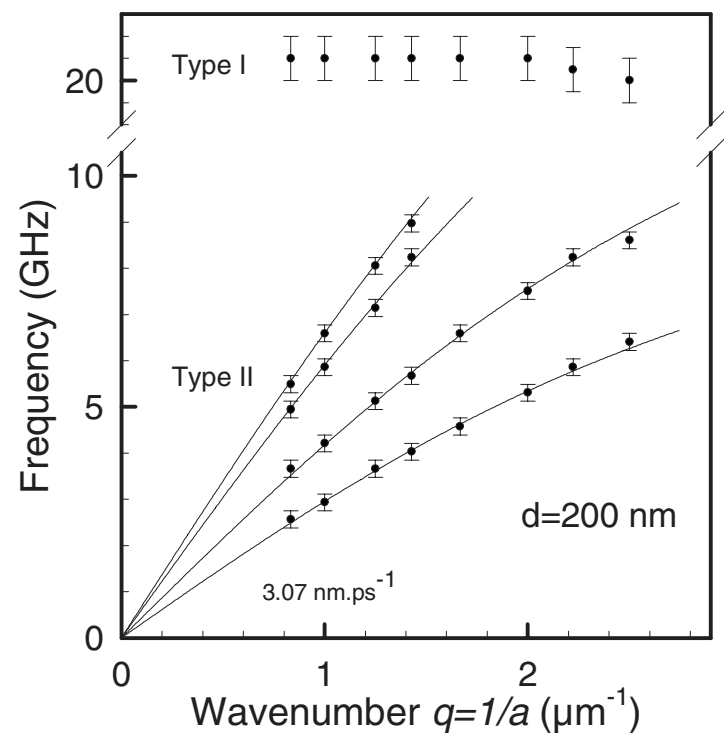

FIG. 5. Frequency of type II modes as a function of the inverse of the lattice constant obtained from the $d=200 \mathrm{~nm}$ cube set. Solid line: modeling using an analytical model described in the text.

In order to determine the role of the probe wavelength $(\lambda)$ in type II detection, we studied all the samples using a red probe then a blue probe. The observation of type II modes is indicated in Table I by an asterisk ( ${ }^{*}$ ). Looking at Table I, we reach two conclusions. One first notices that the type II modes are only detected if the $\lambda / a$ ratio is lower than a value close to 1 . That is the reason why they are not observed in the infrared experiments. Second, the wavelength does not affect the frequency content of type II modes (samples F-H present the same type II frequencies using a blue or a red probe). Provided the $\lambda / a$ condition is satisfied, the type II modes appear and do not change with the wavelength.

The dispersion of type II modes could help in clarifying their nature. Changing the angle of incidence has no impact on the frequencies and, thus, does not permit to explore the reciprocal lattice. A way of building a dispersionlike curve for these modes is to explore a series of $d=200 \mathrm{~nm}$ samples with increasing step sizes $a$. Type II modes are detected using a blue probe for the whole set (samples $\mathrm{C}$ and $\mathrm{I}-\mathrm{O}$ in Table I), as expected from the previous criterion. Each sample gave several frequencies which are reported with respect to the wave number $q=1 / a$ in Fig. 5 .

Type I modes are found close to the same frequency for any lattice parameter. On the contrary, type II modes present a strong dispersion. We first examine the extrapolated frequencies when $q$ gets close to 0 , which corresponds to more and more spaced dots. We note that the curves pass through the origin as expected, since collective modes should disappear when the dots are infinitely separated. Around zero, the lower curve presents a slope $(3.07 \mathrm{~nm} / \mathrm{ps})$ very close to the Rayleigh wave velocity in $\mathrm{Al}\left(c_{R}=3.01 \mathrm{~nm} / \mathrm{ps}\right){ }^{20}$ The other curves are deduced from the lower curve by rescaling it with quantified factors $(\sqrt{2}, 2$, and $\sqrt{5})$. The pump excites several frequencies corresponding to the same mode excited along different directions related to the sample periodicities. The wave vector $k$ of the wave excited in the $(i, j)$ direction sat- 
isfies $\left(k \sqrt{i^{2}+j^{2}}\right) a=2 \pi$, from which we retrieve the rescaling factors. The previous analysis confirms the acoustical nature of the collective modes and reveals that they behave as surface acoustic waves in the limit of small $q$. However, they do not reduce to a surface acoustic wave in the $\mathrm{Al}$ underlayer as testified by the strong deviation observed for realistic $1 / a$ values. We can reproduce such a high dispersion using a simple oscillator model, in which a change in the lattice parameter first affects the mass of the unit cell. Assuming a constant stiffness, we can derive the frequencies $f$ as a function of the wave number $q$ :

$$
f(q)=c \sqrt{\frac{h q^{2}}{h+q^{2} d^{3}}},
$$

where $h$ is the underlayer thickness, $d$ is the dot size, and $c$ is a sound velocity which will be used to fit experimental data. For small values of $q$, this expression reduces to $f(q)=c q$, as expected for acoustic phonon modes. As shown in Fig. 5, such a simple model perfectly reproduces all the experimental data assuming $c=3.07 \mathrm{~nm} / \mathrm{ps}$. The three upper branches are deduced from the first according to the excitation of multiple wave vectors $[(1,0),(1,1),(2,0)$, and $(2,1)]$. Such an agreement finishes with a demonstration that type II modes well originate from an acoustic mode of the periodic struc- ture which propagates in various directions at the sample surface.

The detection of type II modes is based on a different and more efficient mechanism compared to type I. This mechanism for which a laser-wavelength condition has been identified may involve plasmonic effects. Indeed, the nanostructured metallic surface of our samples can couple the incident light to plasmons propagating along the surface. ${ }^{21}$ As shown, type II modes also propagate along the surface, leading to a simultaneous localization of electromagnetic and acoustic fields. This can enhance the acousto-optic interaction, which is consistent with the high efficiency of type II mode detection. ${ }^{22} \mathrm{~A}$ treatment of the plasmonic nature of our sample is needed to investigate quantitatively the role of surface plasmon in type II mode detection, which is beyond the scope of this Brief Report.

In this Brief Report, we coherently excite and detect hypersonic vibrations in nanometric phononic crystals. Two kinds of modes are identified. First, individual vibrations are detected through the photoelastic mechanism. Second, the periodic arrangement of the dots leads to other modes whose frequencies depend on both the dot size and the lattice parameter. A simple elastic model perfectly reproduces the dispersion curve and shows that these collective modes propagate along the sample surface in different directions. Their detection is very efficient and based on a specific mechanism which may involve plasmonic effects.

*arnaud.devos@isen.fr

${ }^{1}$ L. Brillouin, Wave Propagation in Periodic Structures (Dover, New York, 1953).

${ }^{2}$ J. H. Page et al., Phys. Status Solidi B 241, 3454 (2004).

${ }^{3}$ M. S. Kushwaha, P. Halevi, L. Dobrzynski, and B. DjafariRouhani, Phys. Rev. Lett. 71, 2022 (1993).

${ }^{4}$ T. Gorishnyy, C. K. Ullal, M. Maldovan, G. Fytas, and E. L. Thomas, Phys. Rev. Lett. 94, 115501 (2005).

${ }^{5}$ M. H. Kuok, H. S. Lim, S. C. Ng, N. N. Liu, and Z. K. Wang, Phys. Rev. Lett. 90, 255502 (2003).

${ }^{6}$ H. S. Lim, M. H. Kuok, S. C. Ng, and Z. K. Wang, Appl. Phys. Lett. 84, 4182 (2004).

${ }^{7}$ M. Nisoli, S. De Silvestri, A. Cavalleri, A. M. Malvezzi, A. Stella, G. Lanzani, P. Cheyssac, and R. Kofman, Phys. Rev. B 55, R13424 (1997).

${ }^{8}$ C. Voisin, D. Christofilos, N. D. Fatti, and F. Vallée, Physica B 316-317, 89 (2002).

${ }^{9}$ W. Qian, L. Lin, Y. J. Deng, Z. J. Xia, Y. H. Zou, and G. K. L. Wong, J. Appl. Phys. 87, 612 (2000).

${ }^{10}$ Y. Hamanaka, J. Kuwabata, I. Tanahashi, S. Omi, and A. Nakamura, Phys. Rev. B 63, 104302 (2001).

${ }^{11}$ T. D. Krauss and F. W. Wise, Phys. Rev. Lett. 79, 5102 (1997).

${ }^{12}$ M. A. van Dijk, M. Lippitz, and M. Orrit, Phys. Rev. Lett. 95, 267406 (2005).

${ }^{13}$ G. A. Antonelli, H. J. Maris, S. G. Malhotra, and J. M. E. Harper, J. Appl. Phys. 91, 3261 (2002).

${ }^{14}$ H.-N. Lin, H. J. Maris, L. B. Freund, K. Y. Lee, H. Luhn, and D. P. Kern, J. Appl. Phys. 73, 37 (1993).

${ }^{15}$ L. Dhar and J. A. Rogers, Appl. Phys. Lett. 77, 1402 (2000).

${ }^{16}$ D. M. Profunser, O. B. Wright, and O. Matsuda, Phys. Rev. Lett. 97, 055502 (2006).

${ }^{17}$ L. Landau and E. Lifchitz, Theory of Elasticity (Pergamon, New York, 1959).

${ }^{18}$ A. Devos and C. Lerouge, Phys. Rev. Lett. 86, 2669 (2001).

${ }^{19}$ A. Devos and A. LeLouarn, Phys. Rev. B 68, 045405 (2003).

${ }^{20}$ O. L. Anderson, in Physical Acoustics, edited by W. P. Mason (Academic, New York, 1965), Vol. 3B, pp. 43-95.

${ }^{21}$ L. Martín-Moreno, F. J. Garcia-Vidal, H. J. Lezec, K. M. Pellerin, T. Thio, J. B. Pendry, and T. W. Ebbcsen, Phys. Rev. Lett. 86, 1114 (2001).

${ }^{22}$ D. A. Mazurenko, X. Shan, J. C. P. Stiefelhagen, C. M. Graf, A. van Blaaderen, and J. I. Dijkhuis, Phys. Rev. B 75, 161102(R) (2007). 